Mens

revue d'histoire intellectuelle de l'Amérique française

Marie-Thérèse Lefebvre. Rodolphe Mathieu. L'émergence du statut professionnel de compositeur au Québec, 1890-1962. Sillery, Septentrion, 2004. 278 p.

\title{
Caroline Durand
}

Volume 8, numéro 1, automne 2007

URI : https://id.erudit.org/iderudit/1023153ar

DOI : https://doi.org/10.7202/1023153ar

Aller au sommaire du numéro

Éditeur(s)

Centre de recherche en civilisation canadienne-française

ISSN

1492-8647 (imprimé)

1927-9299 (numérique)

Découvrir la revue

Citer ce compte rendu

Durand, C. (2007). Compte rendu de [Marie-Thérèse Lefebvre. Rodolphe Mathieu. L'émergence du statut professionnel de compositeur au Québec, 1890-1962. Sillery, Septentrion, 2004. 278 p.] Mens, 8(1), 144-148.

https://doi.org/10.7202/1023153ar d'utilisation que vous pouvez consulter en ligne.

https://apropos.erudit.org/fr/usagers/politique-dutilisation/ 
Marie-Thérèse Lefebvre. Rodolphe Mathieu. L'émergence du statut professionnel de compositeur au Québec, 1890-1962. Sillery, Septentrion, 2004. 278 p.

Rodolphe Mathieu. L'émergence du statut de compositeur au Québec, 1890-1962 a été récompensé par le Conseil québécois de la musique, qui lui a décerné le prix Opus en 2005 en tant que livre de l'année. La pertinence de cet ouvrage ne fait aucun doute dans le domaine de l'histoire musicale et il contribue utilement au débat sur la modernité culturelle du Québec. L'auteure, Marie-Thérèse Lefebvre, musicologue à l'Université de Montréal, s'est donnée plusieurs objectifs. À travers l'analyse de la carrière d'un musicien et compositeur original, autodidacte et libre-penseur, elle cherche à comprendre comment le statut de compositeur a acquis son autonomie professionnelle. Lefebvre n'hésite pas à placer Rodolphe Mathieu parmi les précurseurs de la modernité en raison de sa philosophie individualiste de la création musicale et de l'importance qu'il accordait à sa fonction de compositeur. Ses idées allaient à l'encontre des tendances clérico-nationalistes qui préconisaient l'inspiration folklorique et visaient l'émergence d'une véritable musique canadienne. Plusieurs intellectuels influents de l'époque, dont Édouard Montpetit, soutenaient aussi une conception utilitariste de la musique et valorisaient peu la composition, préférant soutenir l'interprétation. Marie-Thérèse Lefebvre cherche aussi à expliquer pourquoi Mathieu n'a pas persévéré dans la composition et pourquoi son œuvre fut écartée et oubliée. Ses réponses reposent principalement sur la domination de la pensée nationaliste et sur le poids de la religion catholique, des courants idéologiques peu ouverts à l'individualisme moderne auquel adhérait Mathieu. Réagissant contre les tendances nationalistes et folklorisantes et n'étant appuyé que par un petit cercle d'amis, d'artistes et 
d'intellectuels, Rodolphe Mathieu aurait été victime de son avant-gardisme et déçu du milieu intellectuel et artistique. Les autres explications concernent le manque de moyens de diffusion, les trop grandes exigences commerciales, l'hostilité de la critique, et enfin, l'absence de reconnaissance officielle du statut de compositeur par la société et les institutions de l'époque.

La thèse et les arguments de l'auteure rendent l'ouvrage pertinent pour un lectorat plus large que les seuls spécialistes de l'histoire de la musique. Cependant, il souffre de quelques défauts de structure: certaines parties sont centrées sur le personnage de Mathieu, sa pensée, ses réseaux de sociabilité et sa carrière, alors qu'à d'autres moments, le lecteur a l'impression que Mathieu sert de prétexte à une minutieuse description de l'univers musical et culturel montréalais de la première moitié du XX ${ }^{\mathrm{e}}$ siècle. Si le mélange peut être justifié par la richesse de la contextualisation, il est parfois maladroit, et le lecteur s'interroge sur la pertinence de certains détails. Par exemple, dans le chapitre "Les années de formation », Lefebvre parle de l'enfance et de la famille de Rodolphe Mathieu, ainsi que du monde musical montréalais au début du $\mathrm{XX}^{\mathrm{e}}$ siècle. Plusieurs informations permettent de mieux cerner le personnage : sa formation musicale, sa pensée libérale et athée, sa participation aux débats entre les modernistes et les tenants du folklore, etc. Cependant, nul besoin de connaitre le salaire annuel de l'institutrice de l'école de rang qu'il a fréquentée pour s'imaginer les conditions dans lesquelles il a reçu son instruction primaire. De même, Marie-Thérèse Lefebvre donne souvent des détails d'une lecture fastidieuse au sujet des relations unissant des musiciens canadiens-français et européens, comme des listes de gens présents à certaines soirées ou fréquentant un lieu en particulier, des programmes de concerts, la liste des occupations professionnelles d'un criti- 
que influent, la description des relations entre le pianiste $\mathrm{Al}$ fred Laliberté et le musicien russe Scriabine et entre Rodolphe Mathieu et les membres de la famille Laurendeau, etc. Elle relate aussi quelques débats ayant entouré la création du Conservatoire de musique et de la Société des concerts symphoniques de Montréal. Ces récits insérés dans la biographie et ces listes ne sont pas dénués d'intérêt, surtout pour les spécialistes de l'histoire musicale, qui apprécieront la description de réseaux complexes et l'évocation de longs débats ayant entouré la mise en place d'institutions aujourd'hui considérées comme essentielles à la vie musicale au Québec.

Toutefois, le lecteur ne saisit pas toujours en quoi ces détails contribuent à répondre aux questions soulevées sur le statut de compositeur ou la modernité de Rodolphe Mathieu. Ainsi, le chapitre 4, "Les "retours de Paris" dans le milieu montréalais ", comporte quelques-unes des pages les plus intéressantes du livre, où est décrit le rôle de Rodolphe Mathieu en tant qu'animateur culturel et participant aux débats intellectuels et esthétiques. Cependant, cette partie est précédée d'une mise en contexte sur les années 1920 et de détails sur l'émergence d'une société de musique contemporaine. Elle est suivie d'une assez longue section sur André Laurendeau, évoquant ses études musicales (il fut l'élève de Rodolphe Mathieu) et son désir de faire carrière dans la musique. Ce même chapitre vise aussi à expliquer pourquoi Mathieu a cessé de composer. C'est beaucoup pour un seul chapitre, et l'information aurait pu gagner en clarté si elle avait été organisée autrement : par exemple, plusieurs éléments contextuels auraient pu être regroupés dans un seul chapitre.

La partie qui nous semble la plus importante s'intitule "Mathieu : un animateur culturel au centre des débats ». Pour ceux qui s'intéressent à la vie intellectuelle et culturelle du Québec du début du XX' siècle, il s'agit de la portion la plus 
pertinente de l'ouvrage car les positions artistiques et idéologiques de Mathieu sont au cœur du raisonnement qui en fait un moderne et un marginal. Lefebvre situe Rodolphe Mathieu par rapport au régionalisme, au folklore et au nationalisme. En refusant de se fondre dans ces courants idéologiques et esthétiques, Mathieu est progressivement ostracisé par son milieu. Lefebvre montre ainsi l'existence de relations importantes entre idéologies dominantes, esthétiques valorisées et philosophies de la création légitimées par le milieu intellectuel et artistique. Il y a là une piste prometteuse concernant la construction sociale et intellectuelle d'une culture dite nationale, évoquée à plusieurs reprises dans l'ouvrage. Cependant, ce propos n'est pas soutenu par une analyse très étoffée des idéologies. Il est difficile d'en tenir rigueur à l'auteure, qui n'est pas une spécialiste de l'histoire des idées. Mais il est tout aussi difficile de passer sous silence les faiblesses de l'ouvrage découlant de ce fait. Par exemple, on remarque l'imprécision du vocabulaire désignant la société où évoluait Rodolphe Mathieu : les termes québécois, canadien-français, canadien et montréalais sont tous employés pour désigner le même milieu musical et social, sans que l'on sache si cela vient de l'utilisation simultanée de ce vocabulaire par les acteurs de l'époque. Quant au clérico-nationalisme si fréquemment évoqué, il ne fait pas l'objet d'une description substantielle et d'une contextualisation appropriée. Une imprécision semblable entoure l'usage du mot modernité. Si on apprend que le modernisme de Rodolphe Mathieu était le fruit de son individualisme, de son athéisme et de son libéralisme, ces caractéristiques philosophiques ne sont pas définies avec précision et on se demande si elles étaient propres à tous ceux qui se réclamaient de la modernité au début du $\mathrm{XX}^{\mathrm{e}}$ siècle. Les caractéristiques esthétiques des styles musicaux moderne et traditionnel ne sont pas plus claires. La thèse avancée par 
l'auteur pour expliquer l'ostracisme qui a frappé Rodolphe Mathieu et son œuvre étant en grande partie fondée sur l'affrontement entre le clérico-nationalisme et le modernisme, l'argument est fragilisé par ce manque de précision et de profondeur dans l'analyse des idées et des esthétiques musicales.

Ajoutons finalement que le livre aurait été enrichi par l'ajout d'un disque comprenant quelques pièces de Rodolphe Mathieu et de compositeurs contemporains ayant adopté un style différent du sien. Si les spécialistes de la musique connaissent sans doute les œuvres de Mathieu et des autres compositeurs de son époque, il n'en va pas de même pour tous. Il serait plus aisé pour les néophytes de comprendre en quoi consistait l'originalité et la modernité de la musique de Rodolphe Mathieu en écoutant quelques-unes de ses compositions et en les comparant avec des œuvres d'inspiration plus traditionnelle.

Caroline Durand Département d'bistoire Université McGill

\begin{abstract}
Michael D. Behiels. Canada's Francophone Minority Communities. Constitutional Renewal and the Winning of School Governance. Montréal et Kingston, McGill-Queen's University Press, 2004. $438 \mathrm{p}$.
\end{abstract}

Dans cette monographie, Michael Behiels raconte l'histoire de la conquête de la gouvernance scolaire par les minorités francophones du Canada dans les deux dernières décennies du $\mathrm{XX}^{\mathrm{e}}$ siècle en faisant une lecture serrée des sources disponibles. Il est rare que les historiens s'aventurent sur le 